\title{
Mobilité et immobilité dans le cadre du placement des adolescents, des usages de l'espace en tension
}

\section{Fleur Guy}

\section{(2) OpenEdition}

\section{Journals}

Édition électronique

URL : https://journals.openedition.org/e-migrinter/307

DOI : 10.4000/e-migrinter.307

ISSN : 1961-9685

Éditeur

UMR 7301 - Migrinter

\section{Édition imprimée}

Date de publication : 1 septembre 2013

Pagination : 116-126

ISSN : 1961-9685

\section{Référence électronique}

Fleur Guy, « Mobilité et immobilité dans le cadre du placement des adolescents, des usages de l'espace en tension », e-Migrinter [En ligne], 11 | 2013, mis en ligne le, consulté le 20 mai 2021. URL http://journals.openedition.org/e-migrinter/307 ; DOI : https://doi.org/10.4000/e-migrinter.307 


\title{
$4^{\text {ème }}$ partie : Circulations et périodes d'immobilité forcée
}

\author{
Mobilité et immobilité dans le cadre du placement des \\ adolescents, des usages de l'espace en tension
}

Fleur Guy

L'

hébergement et la prise en charge d'un enfant ou d'un adolescent hors de son domicile familial, dans le cadre d'une mesure de placement, implique avant tout un déplacement dans l'espace. L'étude des modalités et effets de ce déplacement (à moyen et long termes) a donné lieu à plusieurs travaux sur les parcours de placement et les itinéraires des individus au sein du dispositif de prise en charge. Les « parcours de placement » analysés par Émilie Potin montrent ainsi la diversité des configurations possibles. La distinction entre " enfants placés", « enfants déplacés " et « enfants replacés ", témoigne de parcours résidentiels où la prise en charge en famille d'accueil peut alterner avec des retours au domicile familial (Potin, 2009).
Un parcours pouvant être décrit par les acteurs sociaux comme chaotique où les effets stigmatisants du passage dans certains établissements (Jamet, 2010) invitent en outre à interroger les conditions et possibilités d'atteindre l'objectif d'insertion sociale du placement. Les trajectoires résidentielles et familiales de femmes placées à l'adolescence montrent par exemple que la période qui fait directement suite à la fin du placement est souvent difficile, en lien avec l'absence ou la faiblesse de solidarités familiales à un moment où elles sont particulièrement sollicitées par ailleurs (Frechon, 2003). Dans une perspective proche de celle étudiant la mobilité résidentielle et ses impacts sociaux, les parcours de vie d'adolescents placés sont donc mobilisés afin de mettre en évidence les liens entre mobilité (spatiale mais aussi institutionnelle) et insertion sociale. Le terme «parcours» renvoie également aux déplacements effectués plus fréquemment, au quotidien. La mobilité est en effet un concept polysémique au sein duquel on peut distinguer, dans son acception spatiale, la mobilité quotidienne, la mobilité résidentielle, le voyage et la migration, en fonction de la temporalité et de la distance du déplacement (Kaufmann, 2006). Si les parcours de personnes anciennement placées peuvent être analysés a posteriori, la mobilité quotidienne révèle quant à elle les rapports complexes que les adolescents peuvent entretenir avec une prise en charge ellemême multiple. Le placement en établissement, décidé dans le cadre d'une mesure administrative ou judiciaire, est lié à différents contextes institutionnels. Plusieurs 
nuances renvoient, d'une part, au statut du placement (administratif, judiciaire civil ou judiciaire pénal) et d'autre part, à celui de l'adolescent placé. C'est ainsi que le placement concerne les «mineurs isolés étrangers », expression qualifiant de manière juridique "un étranger se disant mineur et qui se trouve sans représentant légal sur le territoire français » (Masson, 2010 : 115). Le placement peut donc être analysé comme une étape du parcours des adolescents, dans le cadre d'une mobilité résidentielle ou migratoire.

L'analyse du placement à une échelle temporelle plus réduite permet également de questionner le rôle de cette prise en charge dans la mobilité, quotidienne cette fois, des adolescents. Contrairement à d'autres structures de placement ou d'incarcération des adolescents, les foyers sont à la frontière entre l'ouvert et le fermé, la distance et la proximité. La mobilité quotidienne est fortement contrôlée tout en étant dans certains cas encouragée, aboutissant parfois à des injonctions paradoxales et à une tension forte entre mobilité et immobilité. Cette tension révèle la manière dont l'espace est mobilisé par les individus qui « font avec la distance, les cadres matériels et les contextes sociaux, au double sens du terme : ils ne peuvent pas ne pas y être confrontés, et ils en usent ou cherchent à le faire, mais dans la mesure de leurs moyens » (Ripoll, 2006). Le recours à la mise en mouvement ou à l'immobilisation, à différentes échelles temporelles, est considéré ici comme une double modalité de ce "faire avec l'espace » (Lussault, Stock, 2010). L'usage parfois simultané de ces deux modalités interroge dans un premier temps les objectifs auxquels elles répondent. En quoi les objectifs du placement permettent-ils de comprendre un usage de l'espace qui peut sembler contradictoire? Comment, face à ces injonctions contradictoires, se construisent les représentations spatiales des adolescents?
Pour répondre à ce double questionnement, une enquête a été menée dans trois Maisons d'Enfants à Caractère Social (MECS), habilitées à la fois par la Protection Judiciaire de la Jeunesse (PJJ) et l'Aide Sociale à l'Enfance (ASE). Ces établissements, plus couramment appelés «foyers", sont localisés dans des communes rurales, périurbaines et urbaines du département du Rhône. Trente entretiens semi-directifs ont été réalisés avec les équipes éducatives et de direction des trois établissements, ainsi qu'avec les acteurs institutionnels du département. Le rapport à l'espace des adolescents a été abordé par l'intermédiaire du Jeu de Représentation Spatiale (JRS) qui, en reconstruisant cet espace leur a permis de répondre à la question «Quels sont les endroits où tu vas souvent?».

Le JRS présente différents atouts dans le cadre de cette recherche. À la différence de la carte mentale, qui nécessite l'acquisition de certaines compétences, notamment graphiques, par les enquêtés, le JRS "n'introduit pas de difficulté spécifique à un groupe d'âge pour communiquer à l'enquêteur une représentation de l'espace » (Ramadier, Depeau, 2010) et permet d'envisager les représentations spatiales d'un échantillon incluant des individus ayant un large écart d'âge. Ce premier intérêt du JRS, lié à la dispersion de l'âge de l'échantillon de notre enquête, se double d'un atout lié aux caractéristiques plus sociales des enquêtés. En effet, une forte proportion d'entre eux ne sont pas scolarisés, ou suivent des cursus particuliers, qui témoignent d'un rapport difficile à la scolarité. Dans ce contexte, l'aspect ludique du JRS, par rapport notamment à la cartographie mentale classique, facilite certainement l'accord des adolescents pour l'entretien, présenté par l'intermédiaire du jeu. Par ailleurs, la simplicité des consignes du JRS garantit également une forte participation de l'enquêté dans le déroulement de l'entretien qui commence par le JRS, puis se poursuit avec des questions sur la perception de la 
prise en charge, notamment concernant les règles de sorties. La récolte des données étant encore en cours, l'échantillon de seize individus présenté ici vise à être complété. Cependant, l'analyse des pratiques spatiales des adolescents au regard des variables du genre et du statut administratif (mineur isolé étranger ou non) est permise par la répartition équilibrée des enquêtés entre les quatre modalités possibles.

\section{Une prise en charge en tension entre mobilité et immobilité}

\section{Le placement, réponse à un risque d'immobilité socio-spatiale}

Dans une perspective historique, le développement des établissements destinés à accueillir des mineurs montre, dès le XIX ${ }^{\mathrm{e}}$ siècle, la dimension spatiale des difficultés identifiées chez les enfants ou adolescents « inadaptés ». Ainsi, à la fin du XIX ${ }^{\mathrm{e}}$ siècle, le courant aliéniste théorise la transmission héréditaire des vices mais aussi la transmission de dégénérescences produites par le milieu. Le milieu mis en cause est social, mais il possède aussi une composante spatiale forte: il s'agit du milieu urbain en pleine expansion suite à la révolution industrielle (Pédron, 2005). Si les causes des difficultés repérées chez les adolescents sont spatiales, les solutions mises en place pour y remédier le sont aussi. Les thèses soutenues par le courant aliéniste justifient un déracinement de l'individu de son milieu d'origine: si un milieu est responsable de l'inadaptation sociale des enfants et des adolescents, leur déplacement dans un autre milieu permettra leur réhabilitation dans la société. L'idée d'une nécessaire rupture avec le milieu d'origine, et donc d'une mise à distance, est alors affirmée (Jablonka, 2010).

Aujourd'hui, malgré les récentes évolutions législatives et la nécessaire prise en compte de la proximité aux familles, la "mise à distance » est toujours utilisée par les travailleurs sociaux. Mentionnée par l'ensemble de ceux que nous avons rencontrés, cette notion est cependant polysémique: la distance peut être psychologique et ne recouvre pas toujours une dimension géographique. Le recours par certains acteurs à la catégorie «jeunes de quartiers » s'accompagne d'une prise en compte socio-spatiale du concept de «mise à distance ». L'usage de cette catégorie territorialisée (Tissot, 2007) révèle une perception négative par les équipes éducatives d'un rapport à l'espace marqué par l'ancrage. Le «quartier» est ainsi présenté par les acteurs institutionnels et les éducateurs comme un espace à risque, ainsi qu'en témoigne un professionnel de la PJJ : Les raisons du placement sont nombreuses, soit que le milieu... le risque de récidive est trop important sur le quartier, le magistrat prononce le placement pour prévenir la récidive (Professionnel PJJ, entretien du 21 novembre 2010) ou une éducatrice d'un des établissements d'enquête: [...] mais après le fait que eux, quasiment tous vivent en quartier...c'est compliqué pour eux, c'est compliqué parce que la tentation est trop, trop, forte. Ils ont accès à tout ce qui peut être manvais an bas de leur immenble, en bas de chez. eux. C'est vraiment un mode de vie différent (Éducatrice, Foyer 2, entretien du 5 mai 2012).

Ce risque est accentué par le rapport spécifique que les adolescents entretiennent avec le quartier, rapport marqué par le sentiment d'appartenance. L'appartenance territoriale des adolescents est souvent associée au terme «quartier». L'ancrage renvoie alors à l'attachement de l'adolescent à un territoire mais aussi à une appartenance plus globale, qui passe par exemple par la maitrise des «codes" du quartier, et à une "culture urbaine », évoquée à plusieurs reprises lors de mes observations. Ce fort ancrage territorial est alors mis en relation avec une pratique de la ville spécifique, marquée par l'immobilité et le rôle central de la «rue » comme espace géographique et social. Les éducateurs mentionnent ainsi «les codes de la rue» ou «la culture du ghetto » en parlant de certains adolescents : Ca devient la rue ici, c'est la rue! Y a des crachats, 
des inscriptions, ça y est, c'est le ghetto du [Nom du foyer]!» Puis, Lui [K.] il ramène la loi du quartier an foyer. C'est la loi du plus fort, les filles sont soumises... (carnet de terrain, Foyer 2, 15 février 2012). Lors d'une discussion sur les raisons de l'échec du placement de $\mathrm{H}$. au Foyer 3, S., éducatrice, explique le fait que H. n'ait pas pu rester au Foyer 3 par son attachement à la "cité»: il n'avait pas les mêmes codes, lui c'était les règles de la rue qu'il connaissait, il était pas prêt pour ce qu'on lui proposait, y avait toujours l'appel de la cité (Carnet de terrain, Foyer 3, 10 avril 2012). L'utilisation du terme "ghetto» et l'évocation du quartier comme un espace ayant ses propres lois font écho aux représentations médiatiques et politiques des «banlieues françaises » qui ont contribué à forger le «mythe des cités-ghettos» dans les années quatre-vingt dix (Wacquant, 1992). Ce discours s'inscrit plus globalement dans une "spatialisation des problèmes sociaux » et dans un contexte où la ville est « le lieu par excellence où il faudrait lire, décrire et soigner les problèmes sociaux» (Poupeau, Tissot, 2005).

C'est dans ce contexte que le placement propose un nouvel ancrage territorial aux adolescents. L'attachement et l'appartenance évoqués dans ces extraits d'entretiens introduisent aussi l'idée d'un territoire porteur d'identité pour les adolescents. Cette identité perçue comme négative, et risquant le cas échéant de mettre en échec le placement, peut alors être un enjeu fort de la prise en charge. L'institution propose dans certains cas un nouveau référentiel d'identification aux adolescents, qu'elle justifie par les bénéfices associés au mouvement, à la mobilité, mis en regard avec les risques associés à une immobilité toujours urbaine: Ça permet de marquer une coupure avec... en plus tout le bénéfice d'être à la campagne où y a...non seulement y'a énormément de supports mais, en plus c'est des supports différents de la ville. C'est souvent des gamins qui ont connu que la ville donc quelque part ça revient à pouvoir leur faire décowvir un ailleurs et powvoir se construire dans un ailleurs, en miroir de quelque chose de différent que ce qu'ils ont connu jusque-là (Educateur, Foyer 3, entretien 25 avril 2012).

Le déplacement a donc un double objectif: rompre avec un lieu dont l'attachement est perçu comme négatif et permettre à l'adolescent de construire un lien avec un nouvel espace. La mesure de placement induit une mobilité résidentielle, avec une installation dans un établissement qui engendre un grand nombre de changements. La découverte d'un nouvel espace peut en effet s'accompagner d'un changement d'établissement scolaire et de la création d'un nouveau réseau de connaissances pour les adolescents. Le placement et les changements qui y sont associés ne recueillent cependant pas toujours l'accord des intéressés, et la mise en place des objectifs du placement passe alors par l'organisation et la gestion de la mobilité quotidienne.

\section{Une gestion contradictoire des déplacements quotidiens}

La rupture socio-spatiale induite par le placement justifie, entre autre, le cadre plus ou moins contraignant dans lequel se déploient les déplacements quotidiens des adolescents. Les règles concernant les sorties à l'extérieur de l'établissement ont ainsi pour objectif de limiter le retour d'un adolescent dans les lieux dont il a été éloigné par le placement. Les adolescents sont soumis à un règlement concernant les horaires de leur sorties. Certains foyers sont équipés de dispositifs spatiaux marquant la clôture avec l'extérieur comme des portails, barbelés ou palissades et des agencements visant à contrôler les sorties. L'ensemble de ces dispositifs montre la volonté, à court et long terme, de maintenir les adolescents dans l'enceinte de l'établissement. À court terme, il s'agit de garantir leur protection en s'assurant qu'ils ne sortent pas après dixneuf heures par exemple. À plus long terme, la présence des adolescents au foyer ou à l'extérieur avec les éducateurs favorise leur ancrage dans le territoire de l'établissement. 
La volonté pour les éducateurs que les adolescents soient présents au foyer s'accompagne parfois d'une mise en mouvement face au risque que peut représenter l'immobilité.

En effet, dans plusieurs situations, la présence des adolescents au foyer est envisagée en termes de risques par les éducateurs: lors de conflits entre adolescents, de moments d'agitation du groupe, ou lorsque les équipes éducatives sont en sous-effectif. L'équilibre entre les adolescents, ou entre les adolescents et les éducateurs est alors remis en cause et la mise en mouvement peut être utilisée par les éducateurs pour rétablir cet équilibre. Interrogée sur sa journée de travail pendant laquelle elle a accompagné plusieurs adolescents à leur domicile parental, une éducatrice explique qu'elle préfère être dans la voiture: Ben en même temps, tu gangrènes pas au foyer et eux... ils gangrènent pas non plus...et vu comme c'était vendredi... Quand je lui demande si les accompagnements étaient prévus, elle me répond: Non, c'était pas prévu, c'était pour vider le foyer, pour apaiser tout le monde (carnet de terrain, Foyer 2, 24 janvier 2012). Les adolescents sont bien conscients de ce fonctionnement. À propos d'une sortie en voiture pour aller acheter des bonbons, un garçon constate ainsi : moi je sais pourquoi elle vous a emmenées, c'était pour que les filles restent pas à la maison, elles allaient tout péter! (carnet de terrain, Foyer 2, 27 avril 2012).

Cette mise en mouvement qui se situe dans l'immédiateté peut aussi intervenir sur des temps plus longs. Les exemples de séjours de rupture, de mise au vert ou séjour d'apaisement, montrent bien ce lien entre mise en circulation d'un adolescent et maintien $\mathrm{du}$ groupe $\mathrm{du}$ foyer. Les interrogations des éducateurs du Foyer 1 face aux "mises au vert» répétées d'une adolescente révèlent cette fonction du séjour de rupture. Une éducatrice m'explique ainsi que l'équipe essaie de trouver plusieurs endroits pour accueillir la jeune fille, parce que quand elle est au foyer, elle a une capacité à faire des bistoires (carnet de terrain, Foyer 1, 11 janvier 2012). Toujours à propos de cette adolescente, un autre éducateur, en réunion, regrette le manque de sens de cette mesure: A part pour sawver le foyer, ça perd son sens (Carnet de terrain, Foyer 1, 31 janvier 2012). La contrainte d'immobilité à l'intérieur de l'établissement peut donc se doubler d'une contrainte de sortie, lorsque la présence d'un adolescent est perçue comme menaçante : on le raccompagne pour éviter d'avoir à le gérer ici [...]. Vu qu'il peut être hyper violent, on prend le parti de le raccompagner pour être sûr qu'il se tire (Carnet de terrain, Foyer 2, 13 mars 2012).

Dans un cas comme dans l'autre, les expressions «sauver le foyer» ou "être sûr qu'il se tire » montrent bien la nécessité de ces mesures pour le maintien même de l'institution. La circulation peut ainsi être utilisée par les éducateurs afin de gérer des tensions au sein de l'établissement. La mise en mouvement contrainte, par l'intermédiaire du séjour de rupture notamment, peut aussi prendre une forme plus souple. L'organisation d'une sortie collective ou le fait de laisser sortir un adolescent en dehors des règles de fonctionnement sont par exemple des moyens d'éviter des situations difficiles, liées notamment au nombre trop important d'adolescents dans le foyer ou à l'agitation $\mathrm{du}$ groupe. On voit alors comment la mobilité est négociée entre adultes et adolescents, et comment la circulation des uns et des autres peut être garante d'un équilibre qui permet le maintien de l'établissement.

Le placement est envisagé dans un double objectif : il s'agit à la fois de proposer un cadre stable et un nouvel ancrage aux adolescents placés, ainsi que de maintenir l'équilibre au sein de l'institution. Or, dans certains cas, la présence des adolescents dans l'établissement (objectif recherché à long terme) est perçue comme un risque pour la stabilité de l'établissement (objectif à court terme). La mise en mouvement, par le biais de la mobilité quotidienne et occasionnelle, 
peut dans ce cas être utilisée pour garantir le maintien de l'institution. La mise en regard des deux échelles temporelles d'usage de l'espace révèle la contradiction possible entre la mise en mouvement comme moyen de réaliser un objectif à court terme, et l'ancrage ou la stabilité recherchés à plus long terme (Figure 1).

Figure $\mathbf{n}^{\circ} \mathbf{1}$ : Un usage de l'espace contradictoire.

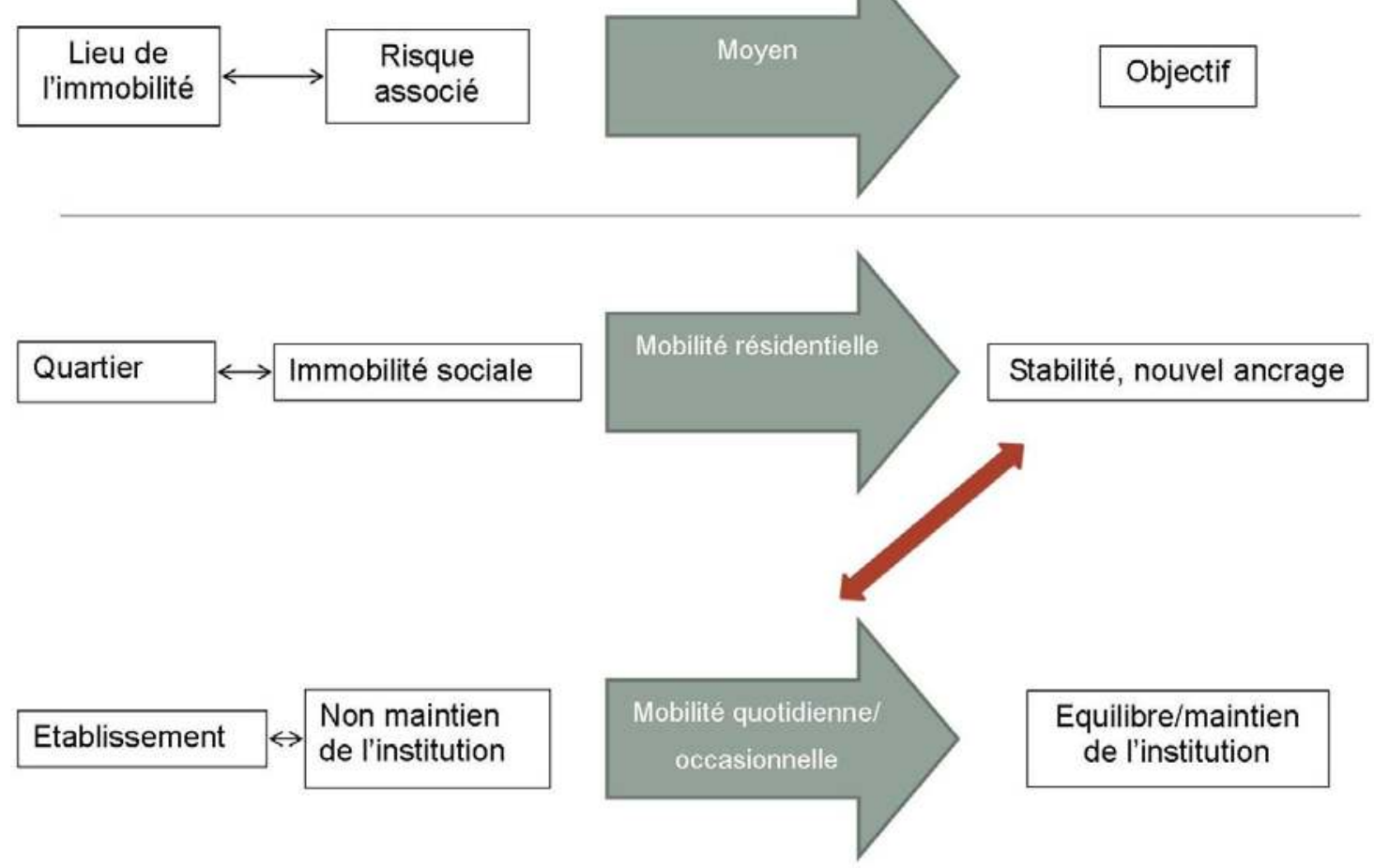

Source : Fleur Guy, 2012.

Face à ces injonctions contradictoires à court et plus long termes, comment se déclinent les pratiques et représentations des adolescents? Si les éducateurs font référence à la rue quand ils parlent des adolescents et de leur ancrage territorial, leurs pratiques et représentations spatiales apparaissent différenciées, notamment pour ce qui concerne l'usage des lieux de circulation. Cette différenciation est appréhendée à travers les caractéristiques sociales des enquêtés (genre) et leur trajectoire résidentielle et/ou migratoire (statut administratif).

\section{Représentation et pratique spatiales : des usages différenciés des lieux de circulation}

Les représentations spatiales issues du JRS peuvent être analysées de différentes manières. Alors que la structure de la représentation donne des indications sur la pratique de l'espace par l'adolescent, les éléments cités permettent d'appréhender les référents spatiaux des enquêtés. Les éléments cités ont ainsi été regroupés en huit catégories, selon une classification fonctionnelle: quartiers, espaces urbains (place, parking...), voies de communication, cours d'eau, espaces commerciaux (magasin, centre commercial, restaurant...), services et équipements (école, gymnase, mairie, poste de police...), espaces verts (parc, aire de jeu...) et bâtiments communs. 
L'objectif de la consigne qui demande à l'adolescent de «montrer les endroits où il va souvent» est de laisser une place importante à l'interprétation de l'enquêté, afin que les diverses situations liées au placement puissent être représentées. La représentation obtenue peut ainsi varier fortement d'un entretien à l'autre, en termes d'échelle ou de nombre d'éléments représentés. Par exemple, les filles représentent en moyenne moins d'éléments que les garçons. Les calculs réalisés à partir des JRS prennent en compte cette variabilité puisque les types d'éléments mentionnés ont été ensuite calculés en pourcentage du total d'éléments cités pour chaque catégorie. Ils permettent de dégager certaines spécificités des représentations spatiales des enquêtés.

L'analyse des représentations spatiales obtenues à l'aide du JRS permet d'identifier des représentations communes ou au contraire propres à certains adolescents. Elle met en avant l'importance de certaines variables dans la compréhension du rapport à l'espace des enquêtés. L'analyse de l'écart de représentation des différentes catégories de lieux en fonction du genre et du statut administratif des adolescents montre dans un premier temps que la majorité des catégories de lieux est représentée uniformément dans les entretiens. Il s'agit des quartiers, des espaces commerciaux, des services et équipements ainsi que des espaces verts. On peut noter un léger écart, en fonction du genre, pour les espaces commerciaux, les services et équipements, et les espaces verts, qui représentent respectivement 15,15 et $10 \%$ des éléments cités par les filles et 11,10 et $7 \%$ par les garçons. En revanche, la répartition est plus équilibrée lorsque l'on prend pour variable le statut administratif de l'adolescent, avec 11, 13 et $9 \%$ pour les mineur(e)s isolé(e)s et 14 , 11 et $8 \%$ pour les non mineur(e)s isolé(e)s. Les adolescents ont ainsi globalement accès aux mêmes lieux, notamment aux équipements et services pouvant être des ressources importantes dans leur parcours d'insertion.

En contrepoint de cette relative homogénéité, deux différences majeures apparaissent dans les éléments cités par les adolescents, concernant les voies de communication et les espaces urbains. Les voies de communication sont tout d'abord surreprésentées dans les JRS réalisés par des mineur(e)s isolé(e)s d'une part et par des garçons d'autre part. Identifié comme un facteur influençant la structure des représentations dans la littérature (Matthews, 1987), le genre n'est pas ici le principal facteur différenciant les adolescents. Les voies de communication représentent ainsi $21 \%$ des éléments cités par les garçons, contre $17 \%$ par les filles. Cet écart qui corrobore les tendances, identifiées chez des individus plus jeunes (Ramadier, Depeau, 2010), selon lesquelles les garçons seraient plus centrés sur les réseaux et les filles sur des points de repère, reste faible par rapport à l'écart entre mineur(e)s isolé(e)s et nonisolé(e)s. Les voies de communication représentent ainsi $31 \%$ des éléments cités par les mineur(e)s isolé(e)s contre $10 \%$ pour les adolescents n'entrant pas dans cette catégorie. Par ailleurs, les représentations sont très différentes au sein d'une même catégorie de genre. L'analyse de la forme des JRS montre bien ces différences: dans certains cas, les différents éléments connectés entre eux, forment des réseaux (Figure 2: JRS, M, 17 ans, mineure isolée). Pour d'autres, l'absence de voies de communication conduit à la représentation d'éléments isolés (Figure 3: JRS, S, 14 ans, non mineure isolée). Plutôt que le genre, c'est bien le statut administratif et l'appartenance de l'adolescent à la catégorie «mineur isolé » qui semble influencer la représentation de voies de communication. 

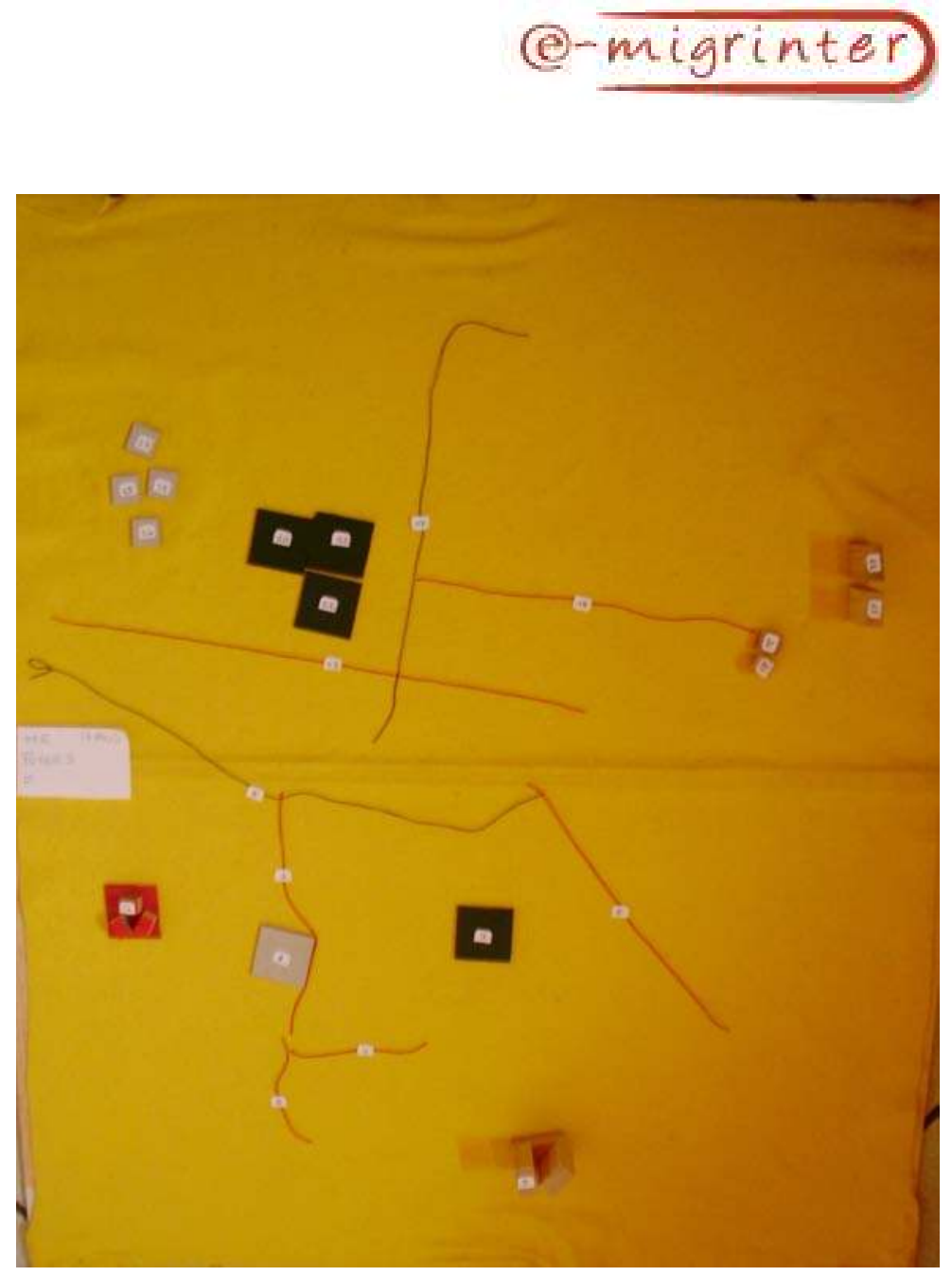

Figure $\mathbf{n}^{\circ} \mathbf{2}: \mathrm{JRS}$ de M, 17 ans, mineure isolée.

Figure $\mathbf{n}^{\circ} 3$ : JRS de S, 14 ans, non mineure isolée.

Source : Fleur Guy, 2012.

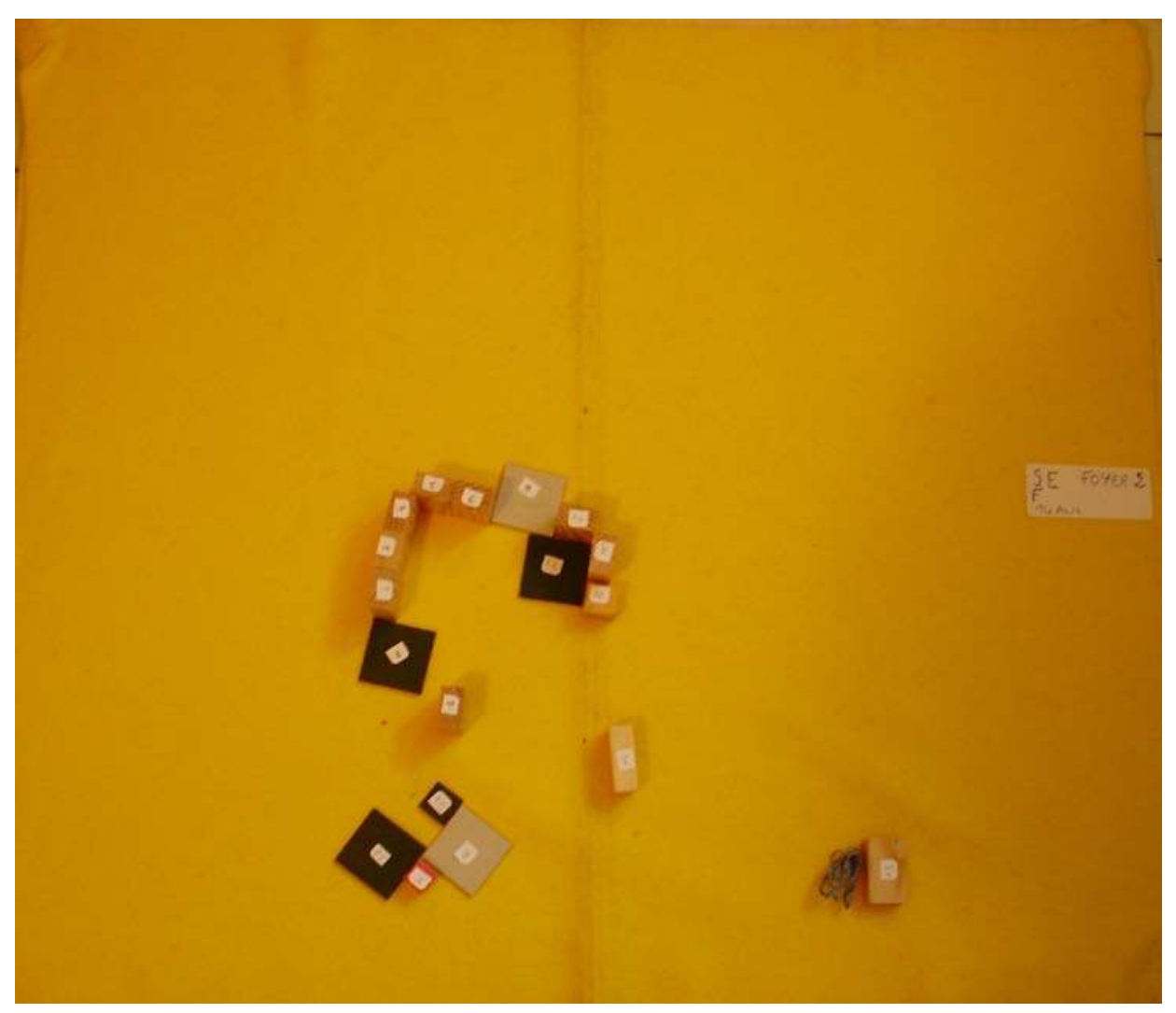


Ces résultats peuvent être mis en relation avec la représentation des espaces urbains dans les JRS. Les éléments utilisés pour représenter des places ou des parkings constituent $16 \%$ des éléments pour les non mineur(e)s isolé(e)s et seulement $7 \%$ dans les JRS réalisés par des mineur(e)s isolé(e)s. Alors que le statut administratif des adolescents ne semble pas influencer l'accès à certains lieux (centres commerciaux, équipements et services, parcs), cette variable révèle une différenciation forte concernant leur mobilité.

Ce constat permet dans un premier temps de relativiser l'influence du genre sur la structuration des représentations spatiales. Il peut, dans un deuxième temps, donner lieu à de multiples interprétations, renvoyant par exemple à la mobilité antérieure connue par les mineur(e)s isolé(e)s et à ses éventuelles conséquences sur la manière de se représenter l'espace, de se déplacer, ou encore à la phase d'apprentissage et de prise de connaissance avec un espace nouveau dans lequel la plupart se trouvent. À ce stade de notre recherche, ces premières interprétations ne peuvent être confirmées. Un échantillon plus important nous permettra en effet de croiser les données présentées ici avec d'autres variables, et notamment celle de la durée du placement au moment de l'entretien ainsi que celle de l'établissement dans lequel l'enquêté est placé.

Si les JRS mettent en avant un écart entre la représentation des lieux de la mobilité et ceux de l'immobilité entre les adolescents, l'analyse des discours permet aussi de compléter ce résultat. Le discours qui accompagne la représentation révèle en effet des pratiques statiques des espaces de circulation. Évoquant le premier élément à gauche de la représentation (Figure $4:$ JRS, E, 16 ans, non mineure isolée), E. explique : c'est la rue, quand on attend quelqu'un avec mes potes!. On retrouve cette pratique immobile des lieux de circulation dans le cas des arrêts de transports en commun, souvent décrits par les adolescents comme des lieux de rencontre et de rendez-vous et n'étant pas intégrés à un réseau dans leur représentation.

Figure $\mathbf{n}^{\circ} 4:$ : JRS de E, 16 ans, non mineure isolée.

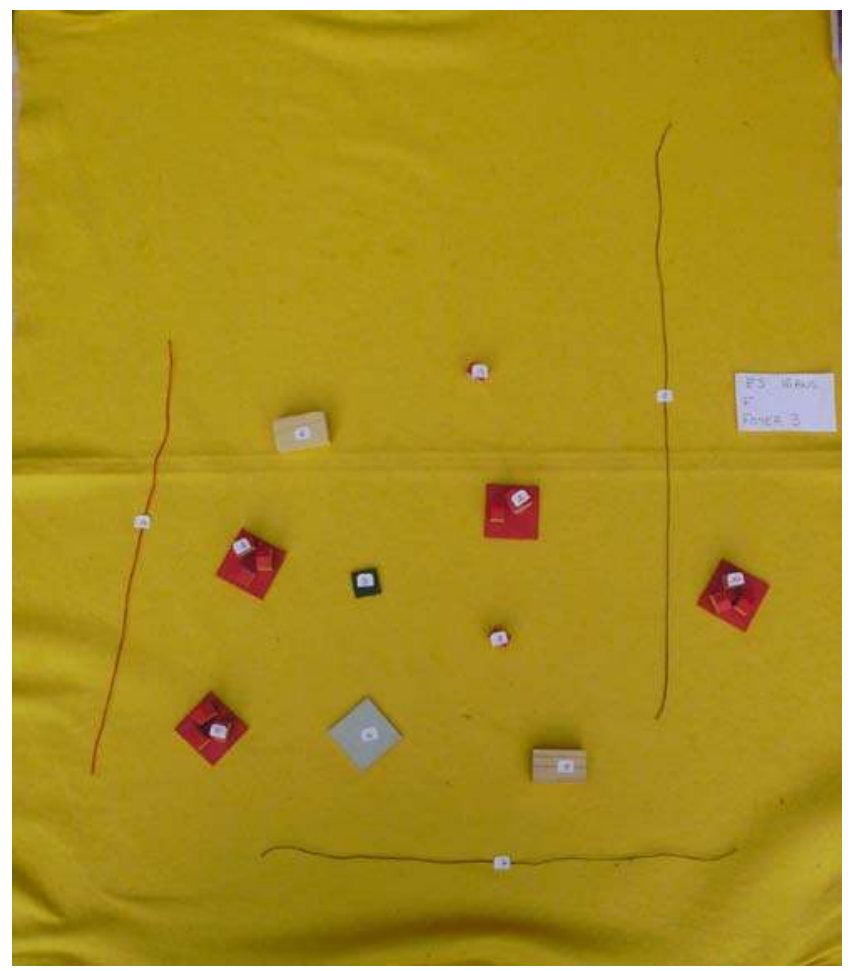

Source : Fleur Guy, 2012 
L'usage de l'espace est au fondement de la prise en charge des adolescents placés. Les différents acteurs du placement mobilisent tour à tour la mobilité et l'immobilité, aboutissant à des injonctions contradictoires selon l'échelle temporelle prise en compte. Si l'ancrage et la stabilité, associés au lieu de placement, sont recherchés à long terme, la circulation et la mise en mouvement sont utilisées pour permettre le maintien de l'institution. Du côté des adolescents, on observe une différence marquée des représentations des lieux de la circulation, en lien avec le statut administratif, révélateur d'un parcours de vie spécifique dans le cas des mineurs isolés étrangers, plus qu'avec le genre. Ces observations nécessitent d'être complétées en prenant en compte le discours des adolescents afin d'analyser les pratiques associées aux différents lieux. Les pratiques immobiles de lieux dédiés à la mobilité laissent entrevoir un usage contradictoire de l'espace par les adolescents. Comme du côté des acteurs sociaux, leurs usages de l'espace révèlent des tensions fortes entre mobilité et immobilité.

Fleur Guy

Doctorante en géographie Université de Lyon (Lumière Lyon 2) UMR 5600 Environnement Ville Société fleur.guy@univ-lyon2.fr

\section{Bibliographie}

Frechon, Isabelle (2003) L'insertion sociale et familiale de jeunes femmes anciennement placées en foyer socio-éducatif, Paris, Université de Paris $10,560 \mathrm{p}$.

Jablonka, Ivan (2010) Les enfants de la République. L'intégration des jeunes de 1789 à nos jours, Paris, Éditions du Seuil, 348 p.

Jamet, Ludovic (2010) Les mesures de placement de mineurs «délinquants » : entre logiques institutionnelles et stigmatisation du public, Sociétés et jeunesses en difficulté, $\mathrm{n}^{\circ} 9$ [en ligne, réf. du 11 janvier 2011] URL : $<$ http:// sejed.revues.org/index6689.h $\underline{\text { tml }}>$.

Kaufmann, Vincent (2006) Motilités, latence de mobilité et modes de vie urbains, in Bonnet, M. ; Aubertel, P. (dir.) La ville aux limites de la mobilité, Paris, Presses Universitaires de France, pp. 223-233.

Lussault, Michel; Stock, Mathis (2010) Doing with space: towards a pragmatics of space, Social Geography [en ligne, réf. du 19 avril 2011] URL : $<$ http://www.socgeogr.net/5/11/2010/sg-5-11-2010.html $>$.

Masson, Bénédicte (2010) «Mineurs isolés étrangers »: le sens d'une appellation, Migrations Sociétés, vol. 22, n¹29-130, pp. 115-128.

Matthews, Michael Hugh (1987) Gender, Home Range and Environmental Cognition, Transactions of the Institute of British Geographers, vol. 12, n¹, pp. 43-56.

Pédron, Pierre (2005) Droit et pratique de la Protection Judiciaire de la Jeunesse, Mineurs en danger, mineurs délinquants, Paris, Gualino, $416 \mathrm{p}$.

Potin, Emilie (2009) Vivre un parcours de placement. Un champ des possibles pour l'enfant, les parents et la famille d'accueil, Sociétés et jeunesses en difficulté, $\mathrm{n}^{\circ} 8$ [en ligne, réf. du 11 janvier 2011] URL: $<$ http://sejed.revues.org/index6428.html $>$.

Poupeau, Franck; Tissot, Sylvie (2005) La spatialisation des problèmes sociaux,_Actes de la recherche en sciences sociales, vol. $4, \mathrm{n}^{\circ} 159$, pp. 4-9. 
Ramadier, Thierry ; Depeau, Sandrine (2010) Approche méthodologique (JRS) et développementale de la représentation de l'espace urbain quotidien de l'enfant, in Danic, I. ; David, O.; Depeau, S. (dir.) Enfants et jeunes dans les espaces du quotidien, Rennes, Presses Universitaires de Rennes, pp. 61-76.

Ripoll, Fabrice; Veschambre, Vincent (2005) Sur la dimension spatiale des inégalités: contribution aux débats sur la " mobilité et le capital spatial », in Arlaud, S. ; Jean, Y.; Royoux, D. (dir.) Rural-urbain, Nouveaux liens, nouvelles frontières, Rennes, Presses universitaires de Rennes, pp. 467483.

Tissot, Sylvie (2007) L'état et les quartiers. Genèse d'une catégorie d'action publique, Paris, Édition du Seuil, 300 p.

Wacquant, Loïc (1992) Pour en finir avec le mythe des cités-ghettos. La différence entre la France et les Etats-Unis, Annales de la Recherche Urbaine, n54, pp. 21-30. 